\section{Military Technical College Kobry El-Kobbah, Cairo, Egypt}

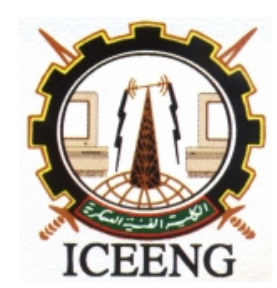

\section{$7^{\text {th }}$ International Conference on Electrical Engineering \\ ICEENG 2010}

\title{
Ballistic Transport in Gate-All-Around Nanowire Transistors
}

\author{
By \\ Dalia Selim Louis* \\ S. H. Gamal* \\ W. F. Farouk* \\ O. A. Omar*
}

\section{Abstract:}

In this paper, we propose a 1D numerical quantum simulator for symmetric gate-allaround nanowire transistors with cylindrical cross section within the effective mass approximation. The simulator is based on a self consistent Schrödinger-Poisson solver, using the finite difference method, in conjunction with a current model assuming ballistic behavior for the transistor. The solutions obtained were first verified analytically when it was available. Electron distribution profiles and I-V characteristics for transistors with different device parameters are numerically evaluated using the proposed simulator. The effects of quantum confinement and low dimensions on these characteristics are indicated.

\section{Keywords:}

Gate-All-Around nanowire transistors, self consistent Schrödinger-Poisson solver, finite difference method and Natori's model.

* Department of Engineering Physics and Mathematics, Faculty of Engineering, Ain Shams University, Cairo, Egypt 


\section{Introduction:}

Recently, Gate-All-Around (GAA) nanowire transistors have become of great interest and are considered a promising candidate for future electronic applications since they improved the gate control and reduced short channel effects [1], [2].

Different materials are considered for growing nanowires. Among these is the silicon which has been studied extensively due to its compatibility with conventional Si CMOS technology [3]. Si nanowires with diameters as small as half nanometer have been grown successfully [4].

In order to understand the physics of the nanowire transistor and to further enhance its behavior, device simulation is necessary. It is worth mentioning that both computational efficiency and high accuracy, taking physical effects into consideration, are crucial in building a simulator.

In this paper, we propose a 1D self-consistent simulator for silicon GAA nanowire transistors based on the effective-mass approximation [5]. A schematic diagram is shown in figure (1), clarifying the structure studied with the used co-ordinate system. The simulator neglects the 2D electrostatics since long channel transistors are assumed (no short-channel-effects are included).

This paper is organized as follows: In section 2, we demonstrate the main flow of the simulator with the basic governing equations. In section 3, the calculated eigenenergies and eigenfunctions are first verified analytically when an infinite potential well is considered. Carrier densities and I-V characteristics are presented while varying different device parameters (diameter and oxide thickness). Finally, section 4 provides a summary for the paper.

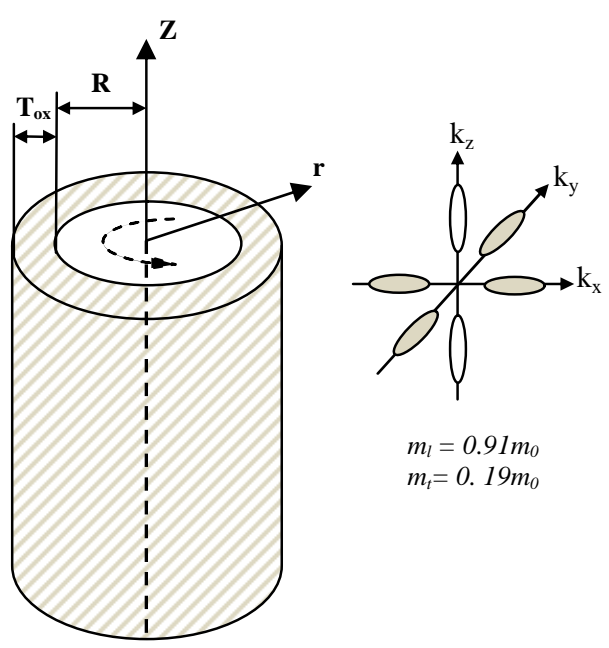

Figure (1): Schematic sketch for the GAA nanowire structure studied in this paper and the constant energy ellipsoids for the silicon in the (100) 


\section{The Simulator and basic equations:}

A flowchart indicating the complete sequence of the solution is shown in figure (2). The calculation involves a self-consistent solution of Schrödinger and Poisson equations. Initially, we set a value for the electrostatic potential that is fed into the Schrödinger equation to determine the carrier density quantum mechanically where the eigenenergies and the eigenfunctions obtained represent the subband energies and the carrier distribution functions respectively. Using the finite difference method (FDM) and the carrier density obtained, we solve the Poisson equation to modify the value of electrostatic potential. This process is repeated until convergence is reached. At this point, the current is calculated through the Natori's ballistic model [6], [7].

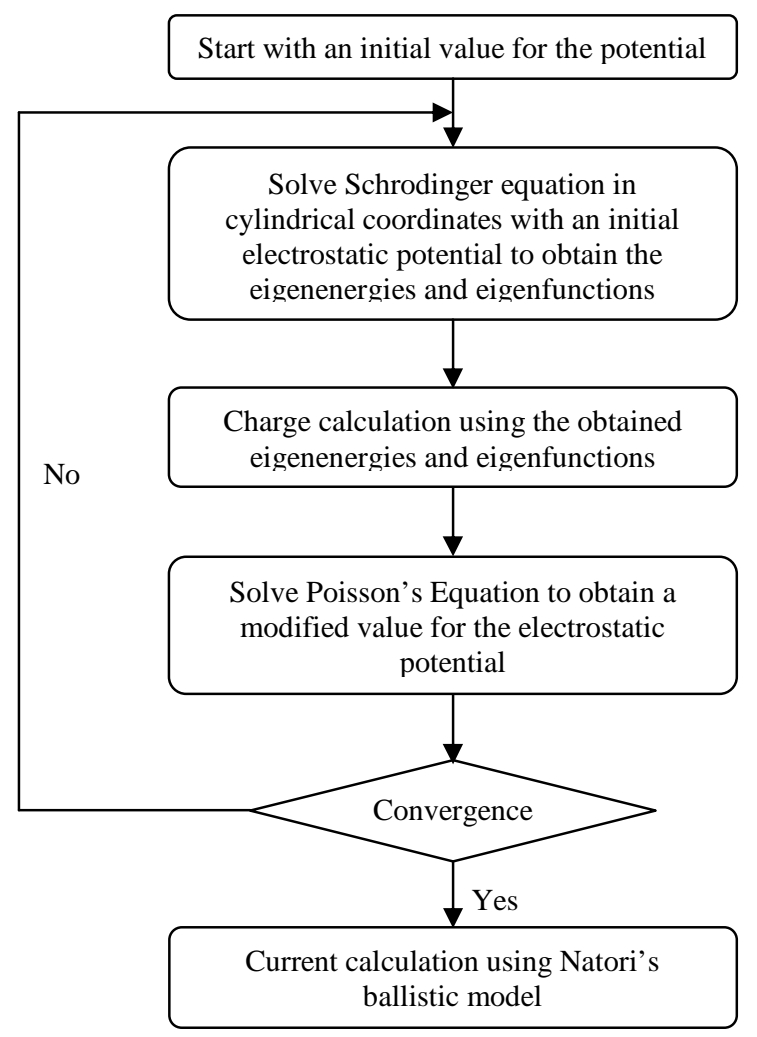

Figure (2): A flowchart for the 1D simulator for GAA nanowire transistors.

\subsection{Schrödinger equation:}

The first step of the quantum solution is obtained by solving the effective mass equation in the confinement directions $(r, \varphi)$ inside the silicon. The 2D Schrödinger equation is given by, 


$$
-\frac{\eta^{2}}{2 \mathrm{~m}_{\mathrm{e}}^{*}} \nabla^{2} \psi(\mathrm{r}, \varphi)-\mathrm{qV}(\mathrm{r}, \varphi) \psi(\mathrm{r}, \varphi)=\mathrm{E} \psi(\mathrm{r}, \varphi)
$$

Where, $\mathrm{E}$ is the energy of the subband (eigenenergy) and $\psi(\mathrm{r}, \varphi)$ is the corresponding wavefunction. The separation of variables method was applied to solve the above equation in which we substituted by,

$$
\psi(\mathrm{r}, \varphi)=\mathrm{R}(\mathrm{r}) \Phi(\varphi)
$$

Since the symmetry is preserved in the $\varphi$-direction and the electrostatic potential is actually a function in the radial distance only, then the $\varphi$-dependent part of the wave equation is solved analytically and gives,

$$
\Phi(\varphi)=\mathrm{e}^{\mathrm{in} \varphi}
$$

Where, $\mathrm{n}$ is the principle quantum number which can only take integer values to maintain the periodicity in the $\varphi$-direction. This reduces the Hamiltonian into

$$
H=-\frac{\eta^{2}}{2 m_{e}^{*}}\left[\frac{d^{2}}{d r^{2}}+\frac{1}{r} \cdot \frac{d}{d r}-\frac{n^{2}}{r^{2}}-q V(r)\right]
$$

Analytical solutions for the reduced Schrödinger equation can be obtained for some restricted forms for the potential [8]. Among these is the simple hypothetical case of zero electrostatic potential which gives an exact solution in the form of Bessel function of the first kind $\mathrm{J}_{\mathrm{n}}(\mathrm{r})$.

In order to solve the equation for a general form for the potential, simple discretization is carried out in the r-direction using finite difference approach. It should be noted that in cylindrical co-ordinates, a singularity problem appears at the point $\mathrm{r}=0$ (at the center of the nanowire). However, the symmetry in the structure restricts the odd order derivatives of the wavefunction to zero at this point thus solving the problem [9].

The wavefunctions are forced to go to zero at the boundary between the silicon and the oxide since no charge is present in the oxide. Solving the wave equation gives rise to the eigenenergies and eigenfunctions. Knowing that the carrier density is proportional to $|\psi|^{2}$, the charge distribution inside the nanowire is calculated according to [7],

$$
\mathrm{n}=\sum_{\mathrm{n}} \mathrm{n}_{\mathrm{L}} \cdot\left|\psi_{\mathrm{n}}\right|^{2}=\sum_{\mathrm{n}}\left(\mathrm{n}_{\mathrm{L}}^{+}+\mathrm{n}_{\mathrm{L}}^{-}\right)\left|\psi_{\mathrm{n}}\right|^{2}
$$


$\mathrm{n}_{\mathrm{L}}$ is the carrier density per unit length for $1 \mathrm{D}$ carrier gas. $\mathrm{n}_{\mathrm{L}}{ }^{+}$and $\mathrm{n}_{\mathrm{L}}{ }^{-}$are the +ve and ve velocity carriers respectively. The summation is carried out over the lowest populated subbands.

$$
\mathrm{n}_{\mathrm{L}}^{+}=\frac{\mathrm{N}_{1 \mathrm{D}}}{2} \mathfrak{J}_{-1 / 2}\left(\frac{\mathrm{E}_{\mathrm{f}}-\mathrm{E}_{\mathrm{n}}}{\mathrm{k}_{\mathrm{B}} \mathrm{T}}\right) \quad \mathrm{n}_{\mathrm{L}}^{-}=\frac{\mathrm{N}_{1 \mathrm{D}}}{2} \mathfrak{I}_{-1 / 2}\left(\frac{\mathrm{E}_{\mathrm{f}}-\mathrm{E}_{\mathrm{n}}-\mathrm{q} \mathrm{V}_{\mathrm{D}}}{\mathrm{k}_{\mathrm{B}} \mathrm{T}}\right)
$$

$\mathfrak{J}_{-1 / 2}$ is the Fermi Dirac integral of order $-1 / 2$ and $N_{1 D}$ is the $1 \mathrm{D}$ effective density of states defined as,

$$
\mathrm{N}_{1 \mathrm{D}}=\frac{\left(2 \mathrm{~m}_{\mathrm{e}}^{*} \mathrm{k}_{\mathrm{B}} \mathrm{T} / \pi\right)^{1 / 2}}{\eta}
$$

\subsection{Poisson equation:}

The first step of the quantum solution is obtained by solving the effective mass equation in the confinement directions $(r, \varphi)$ inside the silicon. The 2D Schrödinger equation is given by,

$$
\frac{\mathrm{d}^{2} \mathrm{~V}(\mathrm{r})}{\mathrm{dr}^{2}}+\frac{1}{\mathrm{r}} \frac{\mathrm{dV}(\mathrm{r})}{\mathrm{dr}}=-\frac{\mathrm{q}}{\varepsilon_{\mathrm{si}}}\left(\mathrm{N}_{\mathrm{b}}+\mathrm{p}-\mathrm{n}\right)
$$

$\mathrm{N}_{\mathrm{b}}$ is the body doping (either acceptors or donors), $\mathrm{p}$ and $\mathrm{n}$ are the hole and electron densities respectively and $\epsilon_{\mathrm{si}}$ is the silicon dielectric constant. For lightly doped silicon nanowires, the hole density and the body doping can be neglected. Thus, equation (8) can be reduced into,

$$
\frac{\mathrm{d}^{2} \mathrm{~V}(\mathrm{r})}{\mathrm{dr}^{2}}+\frac{1}{\mathrm{r}} \frac{\mathrm{dV}(\mathrm{r})}{\mathrm{dr}}=\frac{\mathrm{qn}}{\varepsilon_{\mathrm{si}}}
$$

Poisson's equation is then discretized by means of FDM and solved via NewtonRaphson method such that it should satisfy the following boundary conditions [10],

$$
\left.\frac{\mathrm{dV}(\mathrm{r})}{\mathrm{dr}}\right|_{\mathrm{r}=0}=0 \quad \text { and } \quad \mathrm{V}(\mathrm{r})=\mathrm{V}_{\mathrm{s}}
$$

Where $\mathrm{V}_{\mathrm{s}}$ is the surface potential. 
Applying Gauss' law at the interface point between the silicon and the gate oxide, gives the following relation,

$$
\left.(2 \pi \mathrm{R}) \varepsilon_{\mathrm{si}} \frac{\mathrm{dV}(\mathrm{r})}{\mathrm{dr}}\right|_{\mathrm{r}=\mathrm{R}}=\mathrm{C}_{\mathrm{ox}}^{\prime}\left(\mathrm{V}_{\mathrm{G}}-\phi_{\mathrm{ms}}-\mathrm{V}_{\mathrm{s}}\right) \quad \text { with } \quad \mathrm{C}_{\mathrm{ox}}^{\prime}=\frac{2 \pi \varepsilon_{\mathrm{ox}}}{\ln \left(1+\frac{\mathrm{T}_{\mathrm{ox}}}{\mathrm{R}}\right)}
$$

Where $\mathrm{C}_{\mathrm{ox}}$ ' is the oxide capacitance per unit length.

\subsection{Current Model:}

A semiclassical ballistic transport model (Natori's model) is used to determine the I-V characteristics of the device [6], [7]. In this model the quantum mechanical tunneling from the source to the drain is not considered. The total drain current is obtained by summation over the populated subbands where,

$$
\mathrm{I}_{\mathrm{D}}=\sum_{\mathrm{n}} \mathrm{I}_{\mathrm{n}}
$$

$$
I_{n}=I_{n}^{+}-I_{n}^{-}=\frac{q k_{B} T}{\pi \eta}\left[\mathfrak{I}_{0}\left(\frac{E_{f}-E_{n}}{k_{B} T}\right)-\mathfrak{I}_{0}\left(\frac{E_{f}-E_{n}-q V_{D}}{k_{B} T}\right)\right]
$$

\section{Results and Discussion:}

The solution of the Schrödinger equation using FDM is first verified by comparing the results obtained to the analytical solution in the case of zero electrostatic potential. Figure 3(a) shows an exact agreement between the first four wavefunctions obtained analytically and numerically for a nanowire of radius $3 \mathrm{~nm}$. Note that the free electron mass is used in this simulation. In figure 3(b), the eigenenergies for nanowires of different diameters are plotted; again the conformity between the analytical and numerical energies ensures the validity of the wave equation numerical solution. The eigenenergies are computed analytically according to,

$$
\mathrm{E}_{\mathrm{n}}=\frac{\eta^{2} \mathrm{p}_{\mathrm{nm}}^{2}}{2 \mathrm{~m}_{0} \mathrm{a}^{2}}
$$

$\mathrm{p}_{\mathrm{nm}}$ is the $\mathrm{m}^{\text {th }}$ zero of the $\mathrm{n}^{\text {th }}$ order J-type Bessel function. 
(a)

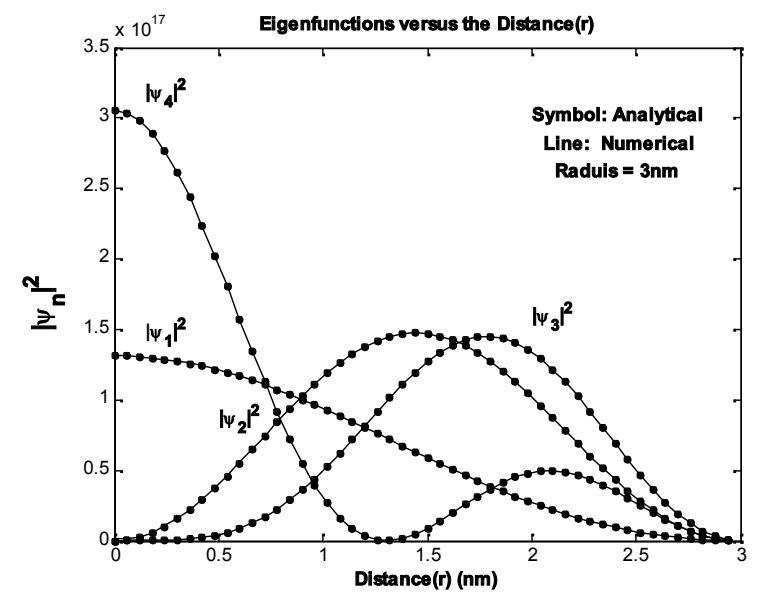

(b)

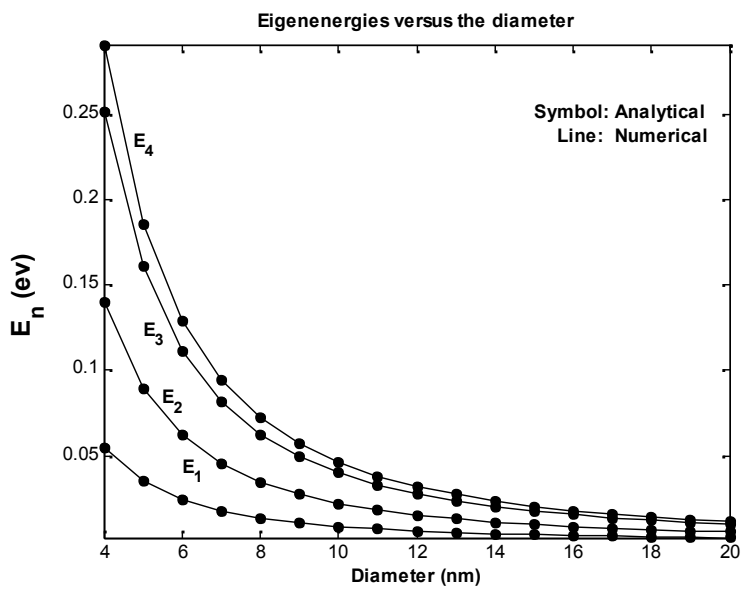

Figure (3): Comparison between the analytical and the numerical (a) wavefunctions and $(b)$ eigenenergies.

To obtain the charge in the confinement direction an approximate isotropic effective mass $\left[2 m_{1} m_{t} /\left(m_{1}+m_{t}\right)\right]$ has been introduced in Schrödinger equation for the fourfold degenerate valleys to preserve the symmetry in the cylindrical coordinates giving rise to the unprimed series of subbands [11] where $\mathrm{m}_{1}$ and $\mathrm{m}_{\mathrm{t}}$ are the longitudinal and transverse effective masses respectively. This mass approximation yields reasonably accurate subband energy levels especially in nanowires with small radii [12]. Another series of primed subbands is obtained by using light effective mass $m_{t}$ for the twofold degenerate valleys.

(a)

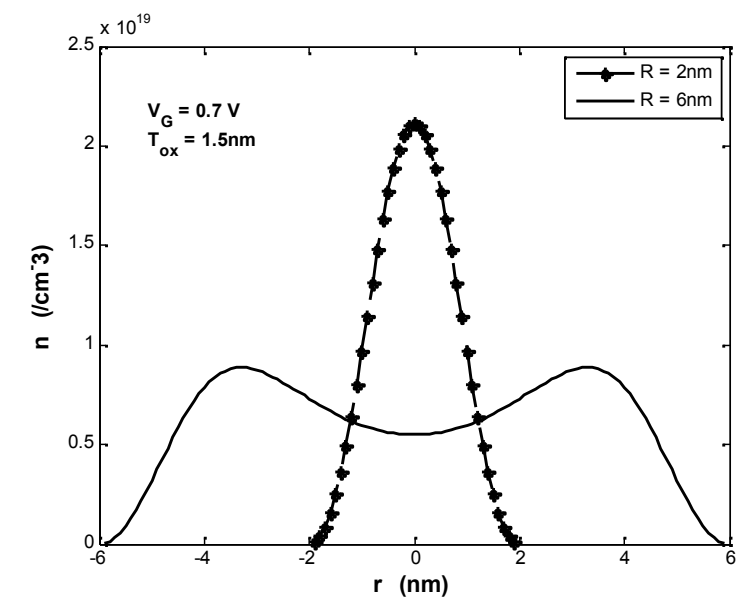

(b)

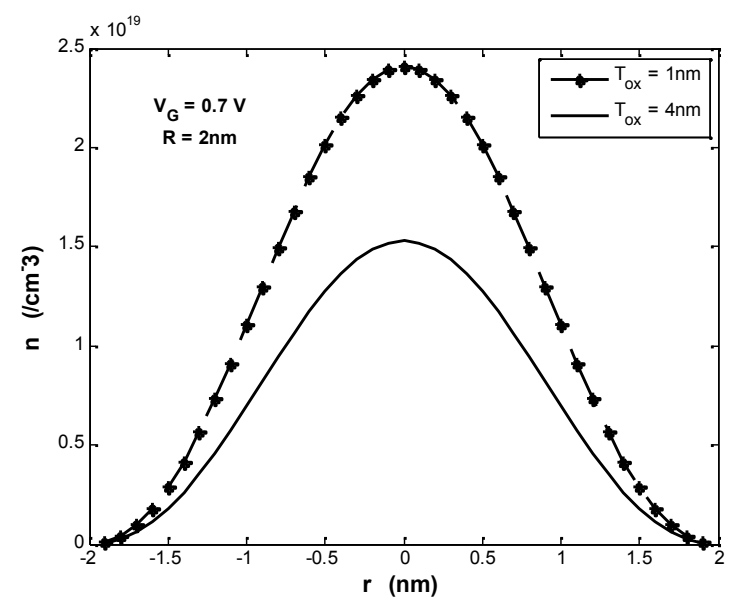

Figure (4): Electron distribution profiles in Si-nanowires with (a) radii $2 \mathrm{~nm}$ and $6 \mathrm{~nm}$ (b) Oxide thickness $1 \mathrm{~nm}$ and $4 \mathrm{~nm}$. 
It is clear from figure 4(a) that decreasing the nanowire diameter confines the inversion charge to the center of the nanowire while in large diameter transistors, most of the inversion charge carriers are confined to regions close to the gate/body interfaces. Increasing the oxide thickness decreases the inversion charge density since most of the gate voltage drops across the oxide (figure 4(b)).

(a)

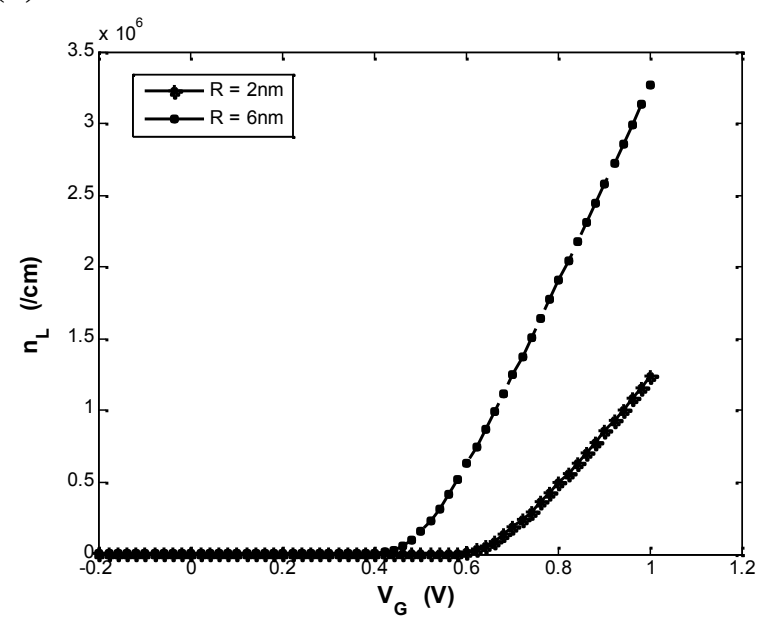

(b)

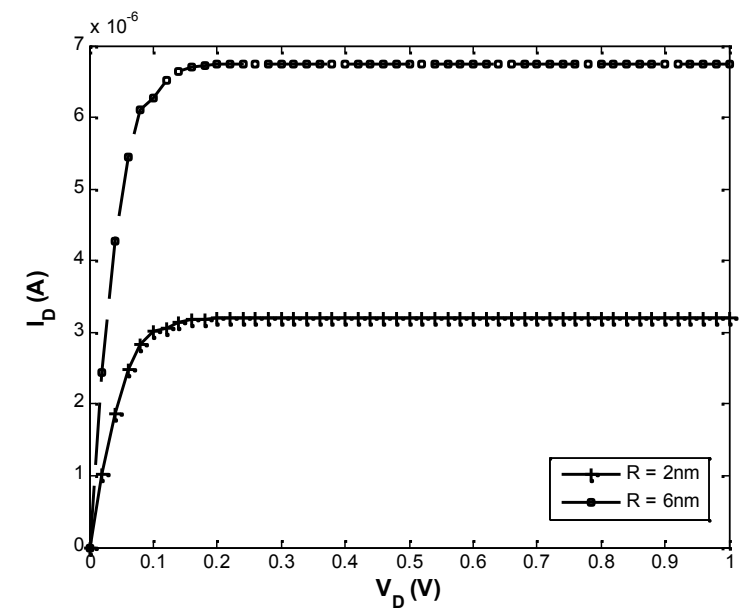

Figure (5): (a) Electron density per unit length as a function of the gate voltage. (b) $I_{D^{-}}$ $V_{D}$ at $V_{G}=0.8 \mathrm{~V}$ for two nanowires of radii $2 \mathrm{~nm}$ and $6 \mathrm{~nm}$ respectively.

Figure 5 indicates that it takes a higher threshold voltage to turn $\mathrm{ON}$ a nanowire device with small diameter than the case of a large diameter device. This is attributable to the size quantization effects leading to increased subband energies in narrow devices. Higher ON current is achieved in case of a wide nanowire.

(a)

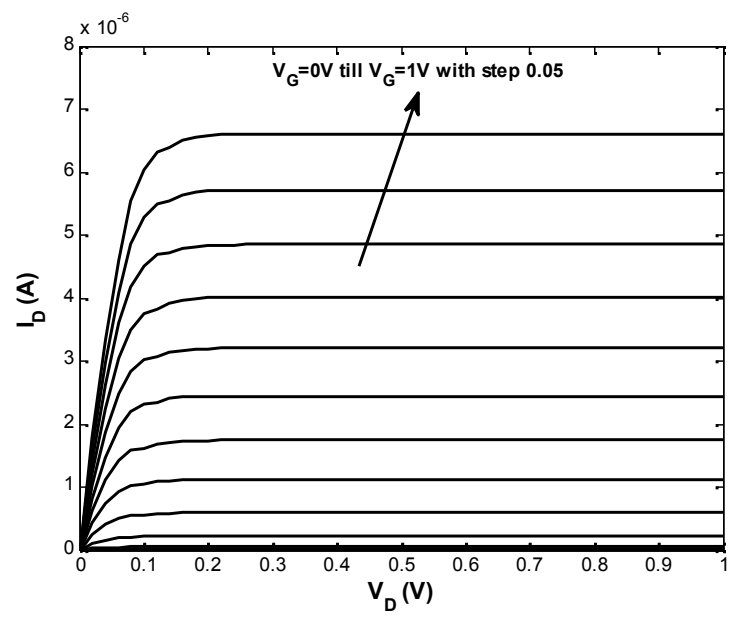

(b)

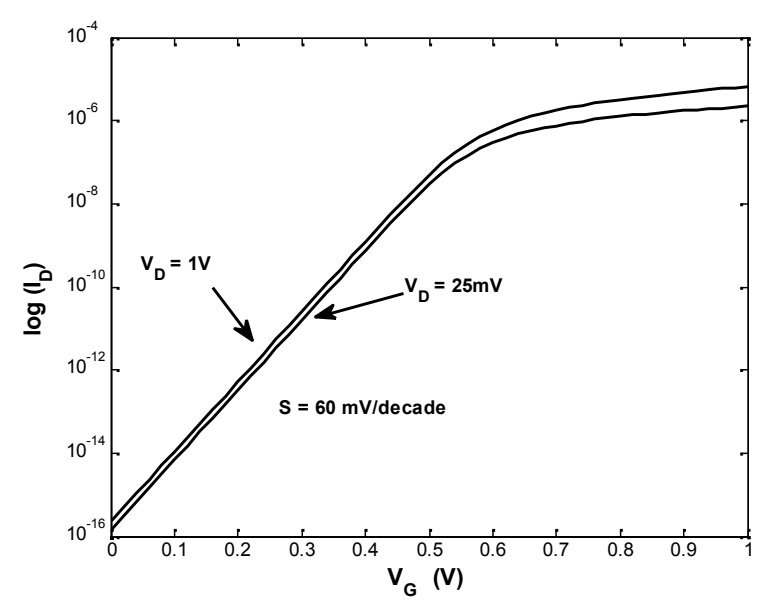

Figure (6): The computed I-V characteristics of a Si-nanowire with radius $2 \mathrm{~nm}$ and oxide thickness $1.5 \mathrm{~nm}\left(\right.$ a) $I_{D} v s . V_{D}$ (b) $\log \left(I_{D}\right) v s . V_{G}$ characteristics. 
The I-V characteristics of a Si-nanowire is shown in figure (6). The simulated device has a radius of $2 \mathrm{~nm}$ and oxide thickness of $1.5 \mathrm{~nm}$. The metal work function is assumed to be equal to the silicon midgap work function and since the body is intrinsic, this leads to zero flatband voltage. It is clear that the transfer characteristics shows an ideal subthreshold swing of $60 \mathrm{mV} /$ decade at low and high drain biases.

\section{Conclusions:}

We have proposed a 1D numerical quantum simulator for symmetric GAA MOSFETs based on a self-consistent Schrödinger-Poisson solver in cylindrical co-ordinates. Subband energies and wavefunctions were evaluated for given geometries and used to obtain the carrier densities. The current was calculated through a semiclassical ballistic model. Comparisons between nanowires with various diameters were made. The solver can be used to simulate nanowires of fabricated from other materials rather than silicon.

\section{References:}

[1] Y. Cui, Z. Zhong, D.Wang,W.Wang, and M. Lieber, High performance silicon nanowire field effect transistors, Nano Lett., vol. 3, pp. 149-152, 2003.

[2] J. Wang, E. Polizzi, And M. Lundstrom, A Computational Study Of Ballistic Silicon Nanowire Transistors, IEDM Tech. Dig., pp. 695-698, Dec. 8-10, 2003.

[3] Y. Wu, Y. Cui, L. Huynh, C. J. Barrelet, D. C. Bell, and C. M. Lieber, Controlled Growth and Structures of Molecular-Scale Silicon Nanowires, Nano Letters, Vol. 4, No. 3, pp. 433-436, 2004.

[4] International Technology Roadmap For Semiconductors, 2009 Edition, Emerging Research Devices

[5] S. Horiguchi, Validity of effective mass theory for energy levels in Si quantum wires, Physica B: Condensed Matter Volume 227, Issues 1-4, Pages 336-338, 1996.

[6] K. Natori, Ballistic metal-oxide-semiconductor field effect transistor, J. Appl. Phys., Vol. 76, No. 8, pp. 4879-4890, 1994.

[7] M. S. Lundstrom and J. Guo, Nanoscale Transistors: Device Physics, Modeling and Simulation. New York: Springer, 2006.

[8] Y. S. Wu and P. Su, Analytical quantum confinement model for short channel gate-all-around MOSFETs under subthreshold region, IEEE Trans. Electron Devices, Vol. 56, No. 11, pp.2720-2725, 2009.

[9] M. N. O. Sadiku, Numerical techniques in Electromagnetics. CRC Press, 2001.

[10] D. Jiménez, B. Iñíguez, J. Suñé, L. F. Marsal, J. Pallarès, J. Roig, and D. Flores, Continuous Analytic I-V Model for Surrounding-Gate MOSFETs, IEEE Electron Device Letters, Vol. 25, No. 8, 2004. 
[11] S. Jin, M. V. Fischetti, and T. W. Tang, Theoretical study of carrier transport in silicon nanowire transistors based on the multisubband Boltzmann transport equation, IEEE Trans. Electron Devices, Vol. 55, No. 11, pp. 2886-2897, 2008.

[12] Y. Yuan, B. Yu, J. Song and Y. Taur, An analytic model for threshold voltage shift due to quantum confinement in surrounding gate MOSFETs with anisotropic effective mass, Solid-State Electronics, Vol. 53, No. 2, pp. 140-144, 2009.

\section{Nomenclatures:}

\begin{tabular}{|c|c|}
\hline \multirow{5}{*}{$\hbar \ldots$} & Planck's constant \\
\hline & Radial direction \\
\hline & Electron effective mass \\
\hline & Electronic charge \\
\hline & Electrostatic potential \\
\hline $\mathrm{r} \ldots$ & Hamiltonian \\
\hline $\mathrm{m}_{\mathrm{e}}^{*} \ldots$ & Fermi level \\
\hline $\mathrm{q} \ldots$ & Subband energy \\
\hline V... & Boltzmann constant \\
\hline H ... & Temprature \\
\hline$E_{f} \ldots$ & Silicon dielectric constant \\
\hline $\mathrm{E}_{\mathrm{n}} \ldots$ & Gate voltage \\
\hline $\mathrm{k}_{\mathrm{B}} \ldots$ & Metal-semiconductor workfunction \\
\hline $\mathrm{T} \ldots$ & Oxide thickness \\
\hline$\epsilon_{\mathrm{si}} \ldots$ & Oxide dielectric constant \\
\hline $\mathrm{V}_{\mathrm{G}} \ldots$ & Drain voltage \\
\hline$\Phi_{\mathrm{ms}} \ldots$ & Drain current \\
\hline $\mathrm{T}_{\mathrm{ox}} \ldots$ & Free Electron mass \\
\hline$\epsilon_{\mathrm{ox}} \cdots$ & Grid spacing in the radial direction \\
\hline$V_{D} \ldots$ & \\
\hline $\mathrm{I}_{\mathrm{D}} \ldots$ & \\
\hline $\mathrm{m}_{0} \ldots$ & \\
\hline a $\ldots$ & \\
\hline
\end{tabular}

